\title{
Magnetostriction of a Superconductor: -Results from the Critical-State Model
}

\author{
Z. Koziol*and R. A. Dunlap \\ Department of Physics, Dalhousie University \\ Halifax, N.S., Canada B3H 3J5
}

August 21, 1995

\begin{abstract}
In many cases, the critical-state theory can be treated as a suffi ciently accurate approximation for the modelling of the magnetic properties of superconductors. In the present work, the magnetostrictive hysteresis is computed for a quite general case of the modified KimAnderson model. The results obtained reproduce many features of the giant magnetostriction (butterfly-shaped curves) reported in the literature for measurements made on single-crystal samples of the hightemperature superconductor $\mathrm{Bi}_{2} \mathrm{Sr}_{2} \mathrm{CaCu}_{2} \mathrm{O}_{8}$. It is shown that addition of a contribution to the magnetostriction in the superconducting state which is of similar origin as in the normal state, offers a broader phenomenological interpretation of the complex magnetostriction hysteresis found in such heavy-fermion compounds as $\mathrm{UPt}_{3}, \mathrm{UR}_{2} \mathrm{Si}_{2}$ or $U B e_{13}$.
\end{abstract}

PACS Numbers: 74.20, 74.70.T 75.80

*electronic addresses: zkoziol@is.dal.ca; http://is.dal.ca/ zkoziol/zkoziol.htm 


\section{Introduction.}

Measurement of the magnetization has been the most commonly used experimental technique for the study of flux- pinning. Recently, however, magnetostriction studies have been shown informative. For example, Ikuta et al. [1] observed giant magnetostriction in $\mathrm{Bi}_{2} \mathrm{Sr}_{2} \mathrm{CaCu}_{2} \mathrm{O}_{8}$ single crystals in the superconducting state and showed that the largest contribution to this effect comes from the interaction between the critical currents induced by changing the external field and the applied field, i.e., the Lorentz force. Much attention has also been directed to dilatometry studies of heavyfermion superconductors. In $U P t_{3}[2]$, the irreversible contribution to the magnetostriction is small but in $U B e_{13}$ [3], $U P d_{2} A l_{3}$ [4] and $U R u_{2} \mathrm{Si}_{2}$ [5], large sample size changes are observed.

In large fields, when the magnetic induction, $B$, changes negligibly across the sample (e.g., in $U R u_{2} S i_{2}$, for $H \geq 10^{3}$ Oe [6]), the idealized situation for a slab of thickness $2 D$ may be written as $B(x) \simeq H-(4 \pi / c) j_{c} \cdot(D-x)$, with $x=0$ at the centre of the slab. Here, $H$ is the external magnetic field and $j_{c}$ is the critical current density, $(4 \pi / c) j_{c}=-d B / d x$. The local Lorentz force exerted on the current carriers, $f=(1 / c)\left(j_{c} \cdot B(x)\right.$, is directed towards the centre of the sample and leads to its compression, for increasing external field. This force must be compensated by the internal local stress, $\sigma(x)$; $\partial \sigma / \partial x=(1 / c) \cdot j_{c} \cdot B(x)$. Hence, with $j_{c}=-(c / 4 \pi) \cdot B / d x$, we find that $\sigma(x)=-\left(H^{2}-B^{2}(x)\right) / 8 \pi$, for the boundary condition $\sigma(D)=0$. For a material characterized by the elastic constant $c_{11}$, the relative change of the sample size, $\Delta D / D$, is given by:

$$
\frac{\Delta D}{D}=\frac{1}{D} \cdot \int_{0}^{D} \frac{\sigma(x)}{c_{11}} d x
$$

which is equal to $-1 /\left(8 \pi c_{11} D\right) \cdot \int_{0}^{D}\left(H^{2}-B^{2}(x)\right) d x$. For the present case, with $(c / 4 \pi) \cdot j_{c} \ll B / D$, we have: $\left(\Delta D / D \simeq-f_{p} /\left(2 \cdot c_{11}\right) \cdot D\right.$. Hence, $\Delta D / D$ is directly related to the flux-pinning-force density, $f_{p}=(1 / c) \cdot B \cdot j_{c}$.

\section{Magnetostriction in the modified Kim-Anderson model}

For qualitative calculations, we shall consider the simplest possible geometry: a slab of thickness $2 D$, with the external magnetic field $H$ applied along its surface. We use a modified Kim-Anderson formula for defining the critical current density, $j_{c}(B)$, corresponding to a local gradient of the 
magnetic induction:

$$
j_{c}=\frac{\alpha}{(B+h)^{n}}=-(c / 4 \pi) \cdot d B / d x .
$$

This dependence unifies many forms of the critical state models. The calculating the magnetization for this geometry and for $j_{c}(B)$ given by Eq. (2) can be found in ref. [7]. This previous work [7] contains an analysis of other effects observed in superconductors which can be explained using the model of a critical-state. The present work provides additional explanation of these phenomena and may be considered as a continuation of our work on this subject.

The calculation of the magnetostriction consists of: $i$ ) finding the magnetic field distribution during the magnetization process, $i i)$ integrating the equation $\partial \sigma / \partial x=(1 / c) \cdot j_{c} \cdot B(x)$ with the boundary condition that $\sigma(D)=0$ and iii) using Eq. (1) for calculation of the magnetostriction. The solution of the equation (2), determines the flux distribution and has the form: $B(x)=( \pm(4 \pi / c) \cdot \alpha \cdot(n+1) \cdot x+\beta)^{1 /(n+1)}-h$, where the integration constant $\beta$ is determined from the boundary condition $B( \pm D)=H$, with the sign of $d B(x) / d x$ dependent on the most recent field change direction occurring at point $x$. For the virgin magnetization curve, $\beta$ is given by: $\beta=(H+h)^{n+1}-H_{m}$, where $H_{m}=\left(H^{*}+h\right)^{n+1}-h^{n+1}$, and $H^{*}$ is the field of the first full flux penetration into the centre of the slab, $H^{*}=\left(\alpha \cdot(n+1) \cdot D+h^{n+1}\right)^{1 /(n+1)}$. Some examples of the flux distribution for different situations are drawn schematically in Fig. 1. In this figure, $B_{0}$ is the magnetic induction in the sample centre and is given in Table 1 for various stages of the magnetization process. The magnetostriction during the virgin magnetization process, for $H<H^{*}$, is found to be;

$$
\begin{array}{r}
\frac{8 \pi c_{11} \cdot \Delta D(H)}{D}=H^{2}-\frac{h^{2}}{H_{m}} \cdot\left((H+h)^{n+1}-h^{n+1}\right) \\
+\frac{(n+1)}{H_{m}} \cdot\left(\frac{(H+h)^{n+3}-h^{n+3}}{n+3}-2 h \cdot \frac{(H+h)^{n+2}-h^{n+2}}{n+2}\right) .
\end{array}
$$

In order to provide the results for the entire magnetization loop in a transparent form, we define the following quantities: 

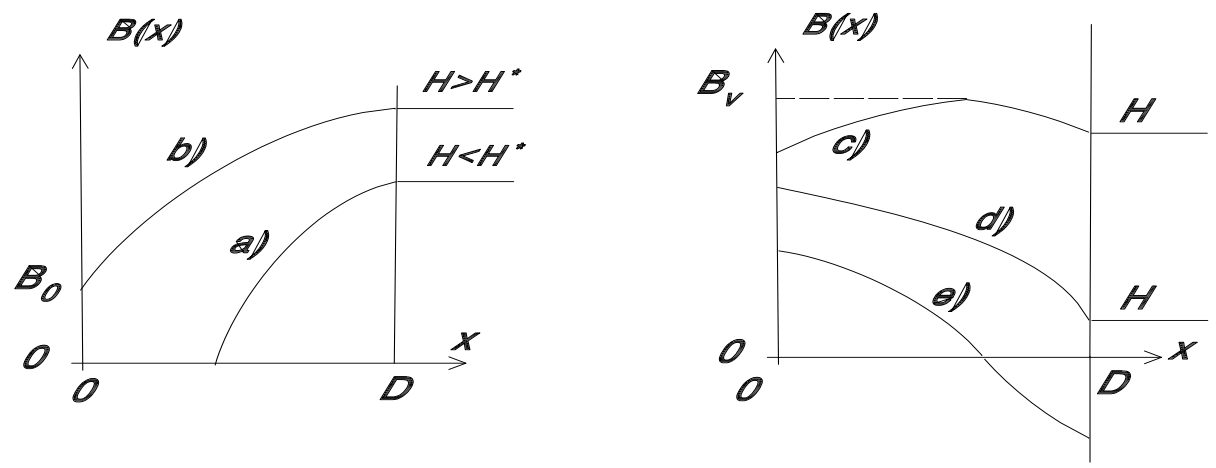

Figure 1: The magnetic induction distribution in a sample volume when a) field increases from 0 to $H<H^{*}$, b) field increases to $H>H^{*}$, c) and $\mathbf{d}$ ) when field decreases from $H_{\max }$ to $H$, and e) when field decreases below $0 . B_{0}$ is the field at the slab centre. $B_{v}$ is the field at the kink of the flux distribution at $x_{v}$, occurring for a certain range of $H$ values after the sweep direction of $H$ is changed. $x_{v}$ is equal to $1-\left(\left(H_{\max }+h\right)^{n+1}-(H+h)^{n+1}\right) /\left(2 \cdot H_{m}\right)$. $B_{v}$ and $B_{0}$ are defined in Table 1.

Table 1. Characteristic fields corresponding to the curves a) to e) in

Fig. 1. $B_{v}$ is equal to $\left(0.5 \cdot\left((H \max +h)^{n+1}+(H+h)^{n+1}\right)\right)^{1 /(n+1)}-h$.

\begin{tabular}{|l|l|l|l|l|}
\hline Case & $H_{0,-}$ & $H_{0,+}$ & $H_{1,-}$ & $H_{1,+}$ \\
\hline a) & 0 & $H$ & 0 & 0 \\
b) & $B_{0}=\left((H+h)^{n+1}-H_{m}\right)^{1 /(n+1)}-h$ & $H$ & 0 & 0 \\
c) & $B_{0}=\left(\left(H_{\max }+h\right)^{n+1}-H_{m}\right)^{1 /(n+1)}-h$ & $B_{v}$ & $B_{v}$ & $H$ \\
d) & $B_{0}=\left((H+h)^{n+1}+H_{m}\right)^{1 /(n+1)}-h$ & $H$ & 0 & 0 \\
e) & $B_{0}=\left(-(|H|+h)^{n+1}+\left(H^{*}+h\right)^{n+1}+h^{n+1}\right)^{1 /(n+1)}$ & 0 & 0 & $H$ \\
\hline
\end{tabular}

$F_{i, 1}\left(H_{i,-}, H_{i,+}\right)=h^{2} \cdot\left(\left(H_{i,+}+h\right)^{n+1}-\left(H_{i,-}+h\right)^{n+1}\right) / H_{m}$,

$F_{i, 2}\left(H_{i,-}, H_{i,+}\right)=-(n+1) /(n+2) \cdot 2 h \cdot\left(\left(H_{i,+}+h\right)^{n+2}-\left(H_{i,-}+h\right)^{n+2}\right) / H_{m}$,

$F_{i, 3}\left(H_{i,-}, H_{i,+}\right)=(n+1) /(n+3) \cdot\left(\left(H_{i,+}+h\right)^{n+3}-\left(H_{i,-}+h\right)^{n+3}\right) / H_{m}$,

with $H_{i,-}$ and $H_{i,+}$ defined in Table 1 , for situations corresponding to those in Fig. 1. The index $i$ enumerates different branches of the magnetic induction curves of continuous $j_{c}(x)$ dependence. Using the above equations, 
the magnetostriction can be written as

$$
\frac{8 \pi c_{11} \cdot \Delta D(H)}{D}=H^{2}-\sum_{i=1,2} f\left(H_{i,-}, H_{i,+}\right),
$$

where

$$
\begin{gathered}
f\left(H_{i,-}, H_{i,+}\right)=S\left(H_{i,-}, H_{i,+}\right) \\
\cdot\left(F_{1}\left(H_{i,-}, H_{i,+}\right)+F_{2}\left(H_{i,-}, H_{i,+}\right)+F_{3}\left(H_{i,-}, H_{i,+}\right)\right) .
\end{gathered}
$$

The function $S\left(H_{i,-}, H_{i,+}\right)$ in Eq. (5) is equal to +1 when $H_{i,-} \leq H_{i,+}$ and to -1 , otherwise. The remaining part of the magnetostriction hysteresis curve, not covered in the Table 1 , may be computed using symmetry: $\Delta D(-H)=$ $\Delta D(H)$.

\section{Discussion}

Some examples of the calculated magnetostriction hysteresis curves are shown in Fig. 2. A large variety of $\Delta D(H)$ dependences is found: from $\Delta D(H)$ proportional to $H$ at $H>H^{*}$, when $n=0$ (Bean model), to $\Delta D(H)$ strongly suppressed by field when $n>1$. For $n>0$, an abrupt passing through $\Delta D(H)=0$ is found, when the field sweep direction changes.
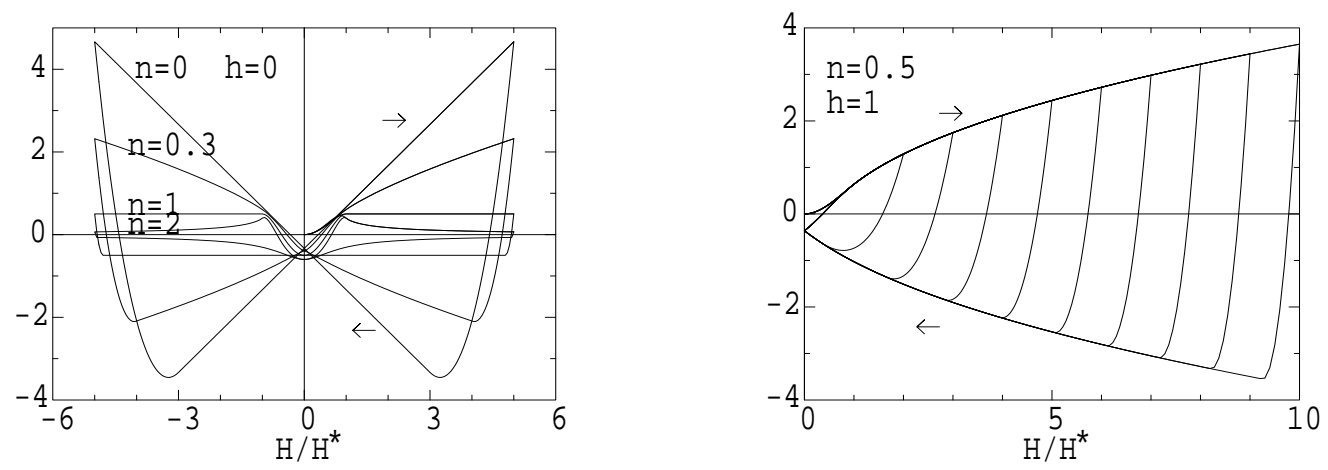

Figure 2: The calculated magnetostriction curves, the virgin one and the entire hysteresis loop; a) $h=0$, while $n$ changes from 0 (the Bean model) to $n=2$, b) when $n=0.5, h=1$ while $H_{\max }$ ranges between 2 and 10 . The vertical scale is in units of $8 \pi \cdot c_{11} \cdot \Delta D / D$. The arrows indicate the direction of field change. 
The field-width of this transition region decreases strongly with an increase of the maximum field applied (Fig. 2b). This is due to a low value of the critical current density at large fields and a decrease in the field change required to reverse the direction of current flow in the sample volume. A significant remanent magnetostriction due to a frozen-in magnetic field is observed. The features discussed above are commonly observed for high- $T_{c}$ and heavy-fermion superconductors.

An unusual effect has been observed recently [5] for single- crystal of $U R u_{2} S i_{2}$. For fields near the upper critical field, $H_{c 2}(T)$, the irreversible contribution to the magnetostriction changes sign compared to the sign observed at lower fields. Moreover, it was observed [5] that the sign of the irreversible contribution to the magnetostriction for some experimental configurations disagred with the prediction of the critical-state model. On the other hand, a large background contribution to the magnetostriction, $\Delta D / D=b \cdot B^{2}$, with the parameter $b$ of the order of $10^{-16} / G^{2}$, has been reported for heavy-fermion materials $[2,3,5]$, which is apparently of the same origin as the normal-state magnetostriction. The role of this contribution on the overall shape of the magnetostriction may be considered. We note that the critical-state magnetostriction component results from the calculation of an integral over the $B^{2}(x)$ dependence. The total magnetostriction in the superconducting state is then assumed to be the sum of the critical-state and the normal- state-like components. The sign of both contributions must be considered carefuly; The critical-state magnetostriction depends on the sign of the magnetic field and current while the normal-state contribution depends on the sign of the coefficient $b$. Using these arguments, we may write:

$$
8 \pi c_{11} \cdot \Delta D(H) / D=H^{2}-\sum_{i=1,2}\left(f\left(H_{i,-}, H_{i,+}\right)-G \cdot\left|f\left(H_{i,-}, H_{i,+}\right)\right|\right),
$$

with $f\left(H_{i,-}, H_{i,+}\right)$ given by Eq. (5) and $G=8 \pi c_{11} \cdot b$. Magnetostriction calculated from Eq. (6) for a few values of the parameter $G$ is shown in Fig. 3. An important feature of these results is that both signs of the magnetostriction and both signs of the irreversible contribution to the magnetostriction hysteresis are possible, while the shape of the curves might only be weakly affected by the presence of a normal-state contribution (except for the presence of a large background proportional to $H^{2}$ ). The hysteresis of the normal-state-like contribution to the magnetostriction in the superconducting state is due to inhomogeneity of the magnetic induction across the 
sample volume, which is caused by the presence of critical current. It is easy to show that at large fields, in particular, near the upper critical field, both contributions to the irreversible magnetostriction are proportional to the critical current density. Hence, the most intriguing property of the magnetostriction in some heavy-fermion superconductors, i.e. the change of sign of the irreversible part of the magnetostriction as a function of the applied field, can not be explained by this model, if it is assumed that the parameter $G$ in Eq. (6) is independant of the magnetic field value.

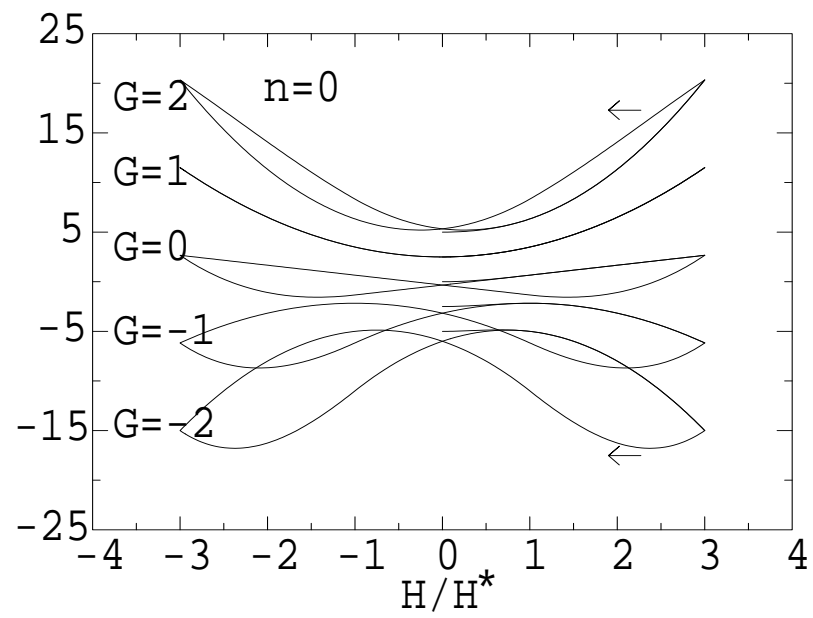

Figure 3: The calculated magnetostriction curves for $n=0$ (the Bean model) for several values of the parameter $G$, determining the normal-state contribution to the magnetostriction: $G=2,1,0,-1$ and -2 , for curves from top to bottom, respectively. One must note that the values of $G$ depend on the units of the magnetic field assumed, which are here equal to $H^{*}$. The vertical scale is in units of $8 \pi \cdot c_{11} \cdot \Delta D / D$. It has been shifted for some curves, for better clarity. The arrows indicate the direction of field change.

Using Eq. (5), magnetic measurements [6] in conjunction with the value of the elastic constant $c_{11}$ allow for a determination of $\Delta D / D$. In $U R u_{2} S i_{2}$, $c_{11}=2.4 \cdot 10^{12} \mathrm{erg} / \mathrm{cm}^{3}[8]$, yields $2 \Delta D / D=3.6 \cdot 10^{-8}$ and $2 \Delta D / D=6$. $10^{-8}$, for $H \| c$ and $H \| a$, respectively, in reasonable agreement with measured properties [5]. On the other hand, for $U P t_{3}$, the elastic constant is larger, 
about $6 \cdot 10^{12} \mathrm{erg} / \mathrm{cm}^{3}[9]$, and the critical current density is much lower [7], resulting in an irreversible contribution to the magnetostriction which is hardly observable experimentally.

Gloos, et al. [4] reported significant difference between the thermalexpansion curves measured during cooling in a magnetic field through the superconducting transition and those measured during heating with the same magnetic field applied at low temperature, for the heavy-fermion superconductor $U \mathrm{Pd}_{2} \mathrm{Al}_{3}$. Any hysteresis of this kind finds its phenomenological explanation within the critical state model and is of the same nature as the magnetization hysteresis in $M_{F C}(T)$ and in $M_{Z F C}(T)$ measurements, as described by us in detail in ref. [7].

4. Conclusions The present calculations have shown that the total magnetostriction in the superconducting state can be separated into criticalstate and normal-state-like components. In general, this approach has provided predictions which are concistent with experimental results in high- $T_{c}$ and heavy-fermion superconductors. In particular, the model allows for the description of both signs of the irreversible contribution to the magnetostriction, though does not allow to explain the mechanism causing the change of this sign as a function of the magnetic field, which is observed in some heavy-fermion systems.

Acknowledgements This work has been made possible by a Killam Postdoctoral Fellowship awarded to Z.K. and by a grant from the NSERC. The initial studies of the subject have been carried out by Z.K. at University of Amsterdam. These have been inspired by the experimental results (some not published yet) of N. van Dijk, A. de Visser, V. Duijn, and have been discussed with them as well with J.J.M. Franse and P.F. de Châtel.

\section{References}

[1] H. Ikuta, et al., Phys. Rev. Lett. 70 (1993) 2166.

[2] N.H. van Dijk, et al, Physica B186-188 (1993) 267.

[3] A. de Visser, et al., Phys. Rev. B45 (1992) 2962. 
[4] K. Gloos, et al., Phys. Rev. Lett. 70 (1993) 501.

[5] N.H. van Dijk, et al., Phys. Rev. B51 (1995) 12665.

[6] Z. Kozioł, et al. J. Magn. Magn. Mat. 140-144 (1994) 2065.

[7] Z. Kozioł, et al., Phys. Rev. B50 (1994) 15978.

[8] P. Thalmeier, et al., Physica C175 (1991) 61.

[9] A. de Visser, Thesis, University of Amsterdam, 1986. 